\title{
MODELLING OF A HYBRID CULTURE SYSTEM WITH A STATIONARY LAYER OF LIQUID PERFLUOROCHEMICAL APPLIED AS OXYGEN CARRIER
}

\author{
Maciej Pilarek*, Katarzyna Dąbkowska \\ Warsaw University of Technology, Faculty of Chemical and Process Engineering, Waryńskiego 1, \\ 00-645 Warsaw, Poland
}

\section{Dedicated to Prof. Ryszard Pohorecki on the occasion of his 80th birthday}

\begin{abstract}
A mathematical model of a hybrid culture system supported with a stationary layer of liquid perfluorochemical (PFC) as a source of $\mathrm{O}_{2}$ for cells which grow in the aqueous phase of culture medium has been developed and discussed. The two-substrate Monod kinetics without inhibition effects, i.e. the Tsao-Hanson equation, has been assumed to characterise the biomass growth. The Damköhler number which relates the growth rate to the mass transfer effects has been used to appraise the regime (i.e. diffusion-limited or kinetics) of the whole process. The proposed model predicted accurately previously published data on the submerged batch cultures of Nicotiana tabacum BY-2 heterotrophic cells performed in a culture system supported with a stationary layer of hydrophobic perfluorodecalin as a liquid $\mathrm{O}_{2}$ carrier. Estimated values of the parameters of the model showed that the process proceeded in the kinetics regime and the growth kinetics, not the effects of the mass transfer between aqueous phase and liquid PFC, had essential influence on the growth of biomass.
\end{abstract}

Keywords: liquid perfluorochemical (fluorocarbon), liquid gas carrier, mathematical modelling, cell/tissue culture, hybrid culture system

\section{INTRODUCTION}

Oxygen is the critical nutrient in all in vitro cultures of those cells which perform aerobic metabolic pathways, i.e. many microbial organisms, as well as all cells isolated from plants, insects and animals, due to the fact that in aerobic metabolism energy is generated by substrate (organic carbon source, mainly) oxidation. Difficulties in achieving a sufficient level of dissolved oxygen, which is close to its physiological range for cells, motivate investigators to search for bioprocess solutions which intensify the mass transfer between the key elements of culture system: an oxygenation agent, a culture medium and continuously metabolizing cells (Garcia-Ochoa et al., 2010; Martin et al., 2010; Suresh et al., 2009).

Water-immiscible and biochemically inert, fully synthetic liquid highly-fluorinated compounds, known as perfluorochemicals (synonym: perfluorocarbons; PFCs), which are characterised by $\mathrm{O}_{2}$ solubility much higher than that in water, are alternatives to conventional aeration systems applied in small-scale bioreactors as well as in mililiter- and microliter-scale cell culture systems (Lowe, 2002; Ntwampe et al., 2010; Pilarek, 2014; Riess 2006). 
There are two ways of liquid PFCs application in submerged culture systems. One of them depends on the flow of an aerated PFC applied as a continuous layer or droplets dispersed in culture medium. From the bioengineering point of view such systems are analogical to typical culture systems with barbotagebased aeration and mathematical models of these bioprocesses have been previously published, e.g. by Amaral et al. (2007), Dragoi et al. (2011) or van Sonsbeek et al. (1992). The second type of PFCsupported cell culture systems utilises a liquid perfluorinated oxygen carrier as a stationary layer in a hybrid culture system developed with two immiscible liquid phases: aqueous-based culture medium and hydrophobic PFC. Such culture systems are exceedingly suitable when a 3-D structure of fragile aggregates, spheroids or sheets of cells, as well as cell-consisting biomaterial-based implant constructs must be preserved to eliminate negative effects caused by hydrodynamic shear forces generated in typical intensively-stirred/agitated culture systems (Davey et al., 2005; Hillig et al., 2014; Pilarek et al., 2013; Pilarek et al., 2014). It should be emphasised that the data available in open literature for such a system are still rather limited, with most quantitative data just omitted.

The aim of our study is to develop and discuss a mathematical model of a hybrid culture system supported with a stationary layer of liquid PFC as a source of $\mathrm{O}_{2}$ for heterotrophic cells which grow in the aqueous phase of culture medium. To our knowledge, this is the first report which quantitatively characterises such type of culture system being increasingly used for cultures of plant and animal cells or tissues, as well as microalgae cells. This is probably also one of the first reports on the modelling of cell culture systems utilising liquid PFCs in general.

\section{MODEL OF A HYBRID CULTURE SYSTEM WITH STATIONARY PHASE OF LIQUID PFC}

\subsection{Scheme of a hybrid culture system}

A scheme of a hybrid culture system supported with stationary layer of liquid PFC taken into consideration in this study is presented in Fig. 1. Cells grow only in the aqueous phase of culture medium with a volume of $V_{\text {aq }}$. The separate phase of liquid PFC has a volume of $V_{\text {PFC. It is assumed }}$ that mixing inside both contacting liquid phases is ideal.

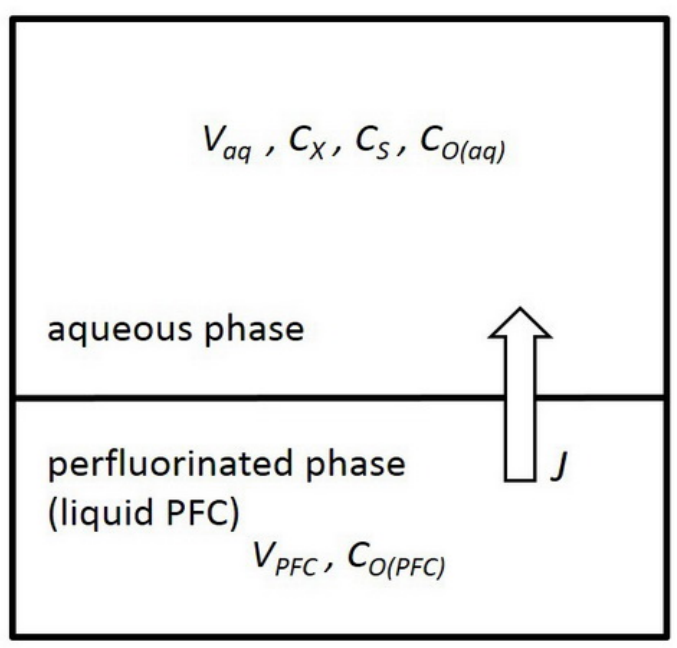

Fig. 1. A scheme of a hybrid culture system supported with stationary layer of liquid PFC

$\mathrm{O}_{2}$ is soluble in a phase of liquid PFC and is transferred into the aqueous phase. The concentration of $\mathrm{O}_{2}$ in the phase of liquid PFC equals to $C_{O(P F C)}$. The growth of cells is limited by two critical factors: concentration of a carbon source $\left(C_{S}\right)$ and concentration of $\mathrm{O}_{2}\left(C_{O(a q)}\right)$ soluble in aqueous phase. 


\subsection{Kinetics and balances}

The rate of mass flux transferred between both liquid, i.e. aqueous and perfluorinated, phases $(J)$ is characterised by the following equation:

$$
J=\left(k_{L} a\right) V_{a q}\left(C_{O(P F C)}-H C_{O(a q)}\right)
$$

where the $\mathrm{O}_{2}$ partition coefficient $(H)$ describes the equilibrium between concentration of $\mathrm{O}_{2}$ dissolved in aqueous and perfluorinated phase:

$$
C_{O(P F C)}^{*}=H C_{O(a q)}^{*}
$$

The cells grow only in the aqueous phase of culture medium. The specific growth rate $(\mu)$ is characterised by the Tsao-Hanson equation, i.e. the two-substrates Monod kinetics is assumed:

$$
\mu=\mu_{\max } \frac{C_{S}}{K_{m, S}+C_{S}} \frac{C_{O(a q)}}{K_{m, O}+C_{O(a q)}}
$$

where:

$$
\begin{gathered}
C_{S} \gg K_{m, S} \\
C_{O(a q)} \gg K_{m, O}
\end{gathered}
$$

as it typically occurs.

The oxygen balance in aqueous phase is characterised by the following equation:

$$
V_{a q} \frac{d C_{O(a q)}}{d t}=\left(k_{L} a\right) V_{a q}\left(C_{O(P F C)}-H C_{O(a q)}\right)-Y_{O X} V_{a q} \mu_{\max } \frac{C_{S}}{K_{m, S}+C_{S}} \frac{C_{O(a q)}}{K_{m, O}+C_{O(a q)}} C_{X}
$$

whilst the oxygen balance in perfluorinated phase is characterised as:

$$
V_{P F C} \frac{d C_{O(P F C)}}{d t}=-\left(k_{L} a\right) V_{a q}\left(C_{O(P F C)}-H C_{O(a q)}\right)
$$

The biomass balance is described as:

$$
V_{a q} \frac{d C_{X}}{d t}=V_{a q} \mu_{\max } \frac{C_{S}}{K_{m, S}+C_{S}} \frac{C_{O(a q)}}{K_{m, O}+C_{O(a q)}} C_{X}
$$

The balance of a carbon substrate, as the growth limiting factor, can be expressed as follows:

$$
V_{a q} \frac{d C_{S}}{d t}=-\frac{1}{Y_{X S}} V_{a q} \mu_{\max } \frac{C_{S}}{K_{m, S}+C_{S}} \frac{C_{O(a q)}}{K_{m, O}+C_{O(a q)}} C_{X}
$$

The initial conditions $\left(t=t_{0}=0\right)$ for Eqs. (6) - (9) are as follows :

$$
\begin{gathered}
C_{O(a q)}=C_{O(a q)}^{*} \\
C_{O(P F C)}=C_{O(P F C)}^{*} \\
C_{X}=C_{X, 0} \\
C_{S}=C_{S, 0}
\end{gathered}
$$

Initial conditions (10) and (11) state that at the beginning of the culture both liquid phases, aqueous as well as perfluorinated, are saturated with $\mathrm{O}_{2}$. 


\subsection{A dimensionless model}

Introducing the following dimensionless variables:

$$
\begin{gathered}
c=\frac{C_{O(a q)}}{C_{O(a q)}^{*}} \\
p=\frac{C_{O(P F C)}}{C_{O(P F C)}^{*}} \\
x=\frac{C_{X}}{Y_{X S} C_{S, 0}} \\
s=\frac{C_{S}}{C_{S, 0}}
\end{gathered}
$$

as well as a dimensionless time:

$$
\tau=t \mu_{\max }
$$

and dimensionless saturation constants:

$$
\begin{gathered}
K_{O}=\frac{K_{m, O}}{C_{O(a q)}^{*}} \\
K_{S}=\frac{K_{m, S}}{C_{S, 0}}
\end{gathered}
$$

the system of balance equations, i.e. Eqs. (6) - (9), results in a dimensionless form:

$$
\begin{gathered}
\frac{d c}{d \tau}=\frac{1}{D a}(p-c)-C_{0} \frac{s}{K_{S}+s} \frac{c}{K_{O}+c} x \\
\frac{d p}{d \tau}=-\frac{1}{D a} \varphi(p-c) \\
\frac{d x}{d \tau}=\frac{s}{K_{S}+s} \frac{c}{K_{O}+c} x \\
\frac{d s}{d \tau}=-\frac{s}{K_{S}+s} \frac{c}{K_{O}+c} x
\end{gathered}
$$

where

$$
D a=\frac{\mu_{\max }}{H\left(k_{L} a\right)}
$$

is the Damköhler number relating the growth rate to the mass transfer rate.

The dimensionless parameter $C_{0}$ defined as:

$$
C_{0}=\frac{Y_{X S} Y_{O X} C_{S, 0}}{C_{O(a q)}^{*}}
$$

may be recognised as the relative initial concentration of the carbon substrate. $C_{0}$ relates the ratio of an amount of $\mathrm{O}_{2}$ assimilated by cells in the case of total carbon substrate consumption and the initial concentration of $\mathrm{O}_{2}$ soluble in aqueous phase of culture medium. 
The dimensionless parameter $\varphi$ relates the volume of aqueous phase to the volume of liquid PFC:

$$
\varphi=\frac{V_{a q}}{H V_{P F C}}
$$

Hereby, $\varphi$ may be recognised as "the relative volume of an aqueous phase" because it is the ratio of a volume of aqueous phase and the volume of water containing the same amount of oxygen as phase of liquid PFC.

Taking onto account the dimensionless variables, the initial conditions (i.e. when $\tau=\tau_{0}=0$ ) for Equations (21) - (24) take the following forms:

$$
\begin{gathered}
c=1 \\
p=1 \\
s=1 \\
x=x_{0}=\frac{C_{X, 0}}{Y_{X S} C_{S, 0}}
\end{gathered}
$$

\section{DISCUSSION OF THE RESULTS}

\subsection{Simulation of the process}

A system of dimensionless Eqs. (21) - (24) has been numerically solved. The classic fourth-order Runge-Kutta method with a constant integration step has been applied to integrate the model equations. Parameters of the simulation were: the Damköhler number $D a$, the relative volume of aqueous phase $\varphi$, the inoculum concentration $C_{X, 0}$ and the relative initial carbon substrate concentration $C_{0}$. The following values of dimensionless saturation constants: $K_{\mathrm{S}}=0.01$ and $K_{\mathrm{O}}=0.1$, have been assumed for calculations. This corresponds to typical bioprocesses, where the initial concentration of carbon substrate is much higher than the saturation constant. In such conditions cells grow with a constant specific growth rate.

The influence of $C_{0}$ on the course of culture has been presented in Fig. 2. $C_{0}$ determines the type of cell growth limitation. For small values of $C_{0}$, the initial concentration of a carbon substrate is the critical factor which limits the final biomass concentration (Fig. 2a). This affects the consumption of a carbon substrate and partially the assimilation of $\mathrm{O}_{2}$. Large values of the parameter $C_{0}$ indicate an excess of a carbon substrate relative to the amount of $\mathrm{O}_{2}$ in a culture system. As a result, $\mathrm{O}_{2}$ is totally assimilated by cells while the consumption of a carbon substrate is only partial (Fig. 2b).

Tu sum up, the critical value of the parameter $\left(C_{0, \text { critical }}\right)$ corresponding to total consumption of both ingredients of a culture medium, i.e. a carbon substrate as well as $\mathrm{O}_{2}$, is defined by the following relationship:

$$
C_{0, \text { critical }}=1+\frac{1}{\varphi}
$$

The influence of $\varphi$ on the cell growth has been presented in Fig. 3. Results of a model calculations presented here, refers to value of $C_{0}$ equal to 5.0, i.e. to the case of the cell growth limited by $\mathrm{O}_{2}$ concentration in the culture system.

According to the theoretical predictions and our experimental results published previously (Pilarek and Szewczyk, 2008; Sykłowska-Baranek et al., 2013), increasing the volume of perfluorinated phase, i.e. 
decreasing $\varphi$, leads to increased biomass growth. In the case of low values of $C_{0}$, i.e. low initial concentration of carbon substrate, the ratio of aqueous phase to perfluorinated one has low impact on the course of culture.

The courses of cultures shown in Fig. 2 and Fig. 3 refer to small values of the Damköhler number, i.e. to the situation when the growth rate is lower than the rate of mass transfer between aqueous phase and liquid PFC. The course of culture in the case of large value of the Damköhler number has been presented in Fig. 4.

a)

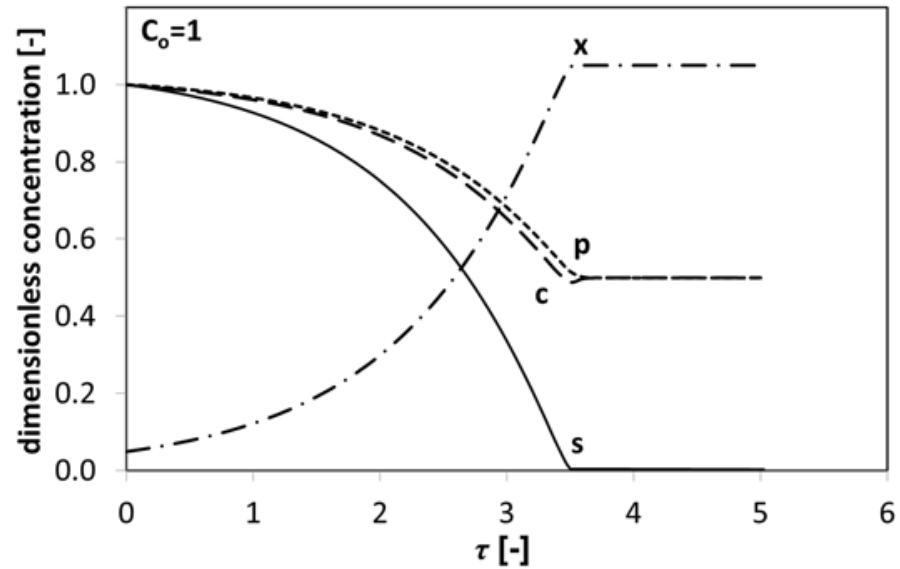

b)

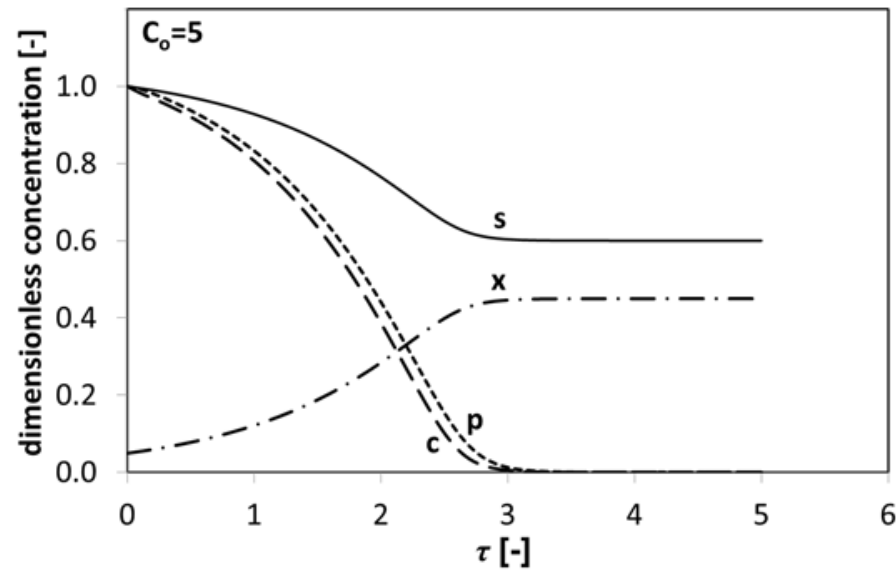

Fig. 2. The influence of the relative (dimensionless) initial substrate concentration $\left(C_{0}\right)$ on the course of culture:

$C_{0}=1$ (a) and $C_{0}=5(\mathrm{~b})$, where: $\mathrm{c}$ - dimensionless concentration of $\mathrm{O}_{2}$ in aqueous phase, $\mathrm{p}$ - dimensionless concentration of $\mathrm{O}_{2}$ in perfluorinated phase, $\mathrm{s}$ - dimensionless concentration of carbon substrate, $\mathrm{x}$ - dimensionless concentration of biomass

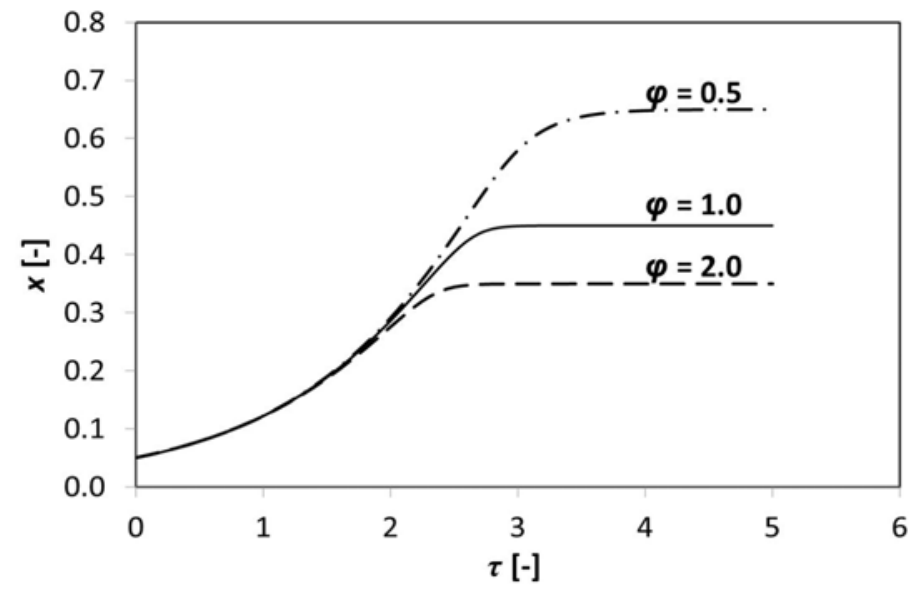

Fig. 3. The influence of the relative volume of aqueous phase $(\varphi)$ on the cell growth 


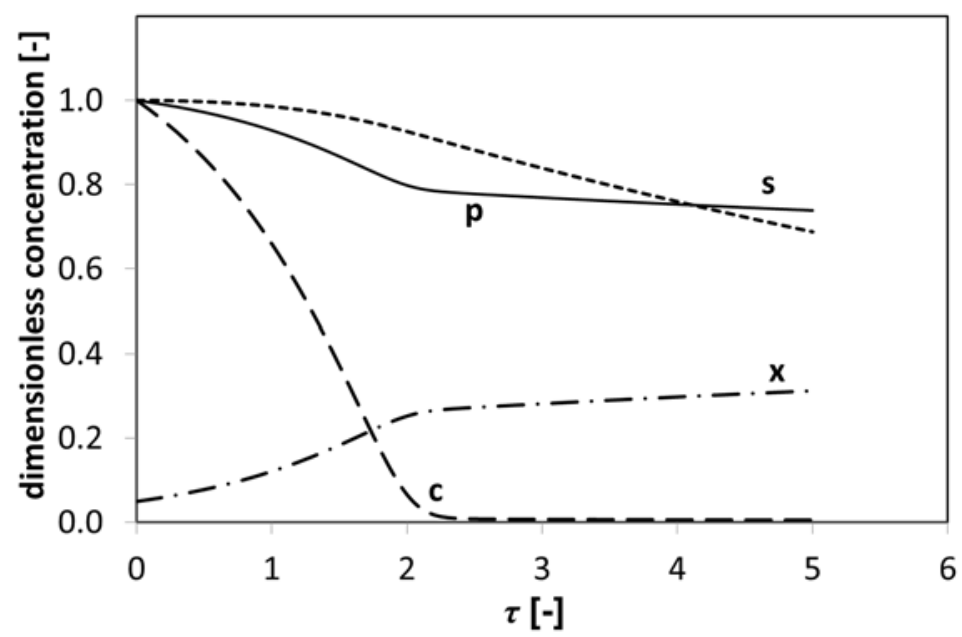

Fig. 4. The simulation of the course of culture for large value of the Damköhler number $(D a=10)$, where: $\mathrm{c}$ - dimensionless concentration of $\mathrm{O}_{2}$ in aqueous phase, $\mathrm{p}$ - dimensionless concentration of $\mathrm{O}_{2}$ in perfluorinated phase, $\mathrm{s}$ - dimensionless concentration of carbon substrate, $\mathrm{x}$ - dimensionless concentration of biomass

If the process proceeds in the diffusion-limited regime, i.e. the mass transfer resistances have significant influence and cannot be neglected, two characteristic stages of a culture are clearly observed. First of them is the exponential growth of cells, during which the assimilation of $\mathrm{O}_{2}$ takes place, and the rate of cell growth depends on the growth kinetics. When the concentration of $\mathrm{O}_{2}$ in aqueous phase is reduced considerably, the rate of biomass growth decreases and further growth of cells is limited by the rate of $\mathrm{O}_{2}$ transfer from liquid PFC into the aqueous phase of culture medium.

The curves of the cell growth for various values of the Damköhler number have been presented in Fig. 5. The influence of the values of the Damköhler number lower than 0.1 on the course of culture is minor. However, the increase in the value of this parameter, e.g. above 1.0, reduces significantly the rate of cell growth.

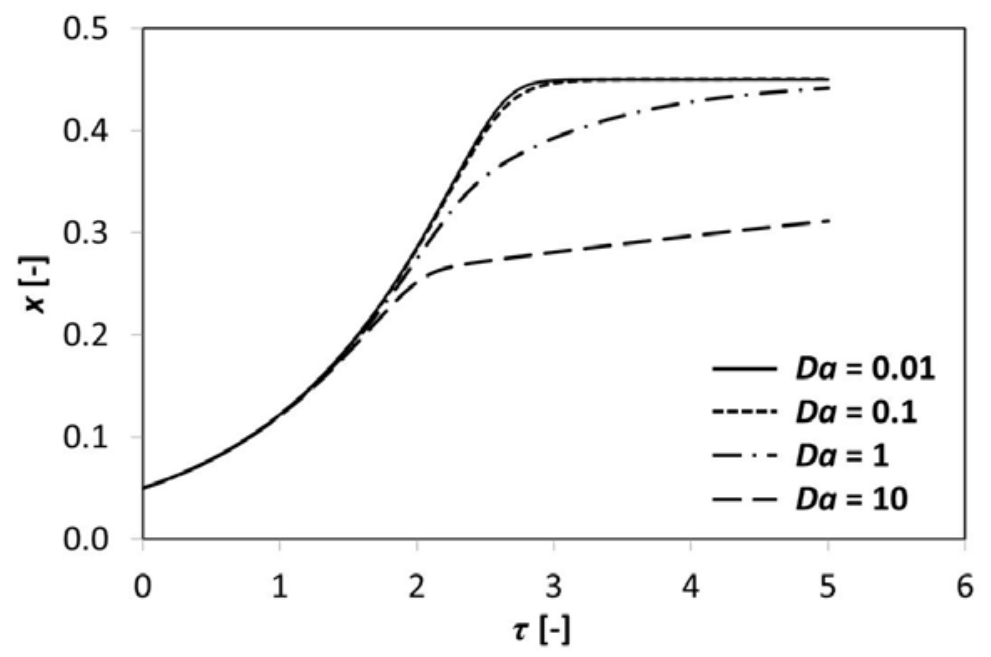

Fig. 5. The influence of the Damköhler number $(D a)$ on the cell growth

\subsection{Estimation of values of the model parameters for a plant cell culture}

Estimation of values of the model parameters was performed for the submerged batch cultures of Nicotiana tabacum BY-2 heterotrophic cells supported with the stationary layer of liquid PFC, based 
on data which have been previously published by Pilarek and Szewczyk (2008). The relevant culture system contained $100 \mathrm{~cm}^{3}$ of culture medium and was supported with air-saturated hydrophobic perfluorodecalin $\left(\mathrm{C}_{10} \mathrm{~F}_{18}\right)$ as the liquid carrier of $\mathrm{O}_{2}$, and volume of liquid $\mathrm{PFC}$ ranged from $20 \%$ to $60 \%$ of aqueous phase volume. The value of the $\mathrm{O}_{2}$ partition coefficient for the water perfluorodecalin system, $H=16.1$, has been calculated based on the values of $C_{O(a q)}^{*}=2.2 \mathrm{mM}$ and $C_{O(P F C)}^{*}=35.3 \mathrm{mM}$. Hence, the relative volume of aqueous phase $\varphi$ varied in the range of $0.1-0.3$.

Values of the Damköhler number for growth of BY-2 cells have been calculated based on $k_{\mathrm{L}} a$ and $\mu$, which have been determined previously (Pilarek and Szewczyk, 2008). The value of the volumetric liquid-side mass transfer coefficient was close to $0.5 \mathrm{~h}^{-1}$, and the specific growth rate of BY-2 cells was from $0.023 \mathrm{~h}^{-1}$ to $0.043 \mathrm{~h}^{-1}$. Therefore the values of the Damköhler number vary from $0.28 \times 10^{-3}$ to $5.2 \times 10^{-3}$. Thus, rather low values of $D a$ mean that the process proceeded in the kinetics regime.

Based on the previously published values of the yield of biomass from substrate coefficient $\left(Y_{X S}\right)$ and yield of oxygen consumption by unit of biomass coefficient $\left(Y_{O X}\right)$, which equal $0.5 \mathrm{~g} / \mathrm{g}$ and $0.3 \mathrm{mmol} / \mathrm{g}$ respectively, as well as taking into account the initial concentration of sucrose, i.e. the carbon substrate, in culture medium which was from 20 to $30 \mathrm{~g} / \mathrm{dm}^{3}$, the value of the dimensionless, i.e. relative, initial concentration of carbon substrate $C_{S, 0}$ has been determined as equal to $1.36-2.05$.

\section{CONCLUSIONS AND OUTLOOK}

The proposed model, based on the two-substrate Monod kinetics without inhibition effects, predicts accurately previously published data on the cultures of $N$. tabacum BY-2 cells performed in a hybrid culture system with a stationary layer of liquid PFC. Estimated values of the model parameters clearly show that the process proceeded in the kinetics regime. The growth kinetics, not the effects of the mass transfer between aqueous phase and liquid PFC, had essential influence on the growth of biomass.

However, for full identification of growth kinetics of plant cell/tissue submerged cultures, some other factors characterising other gaseous compounds should be discussed. Not only $\mathrm{O}_{2}$ dissolved in culture medium has crucial influence on the process, but also concentrations of such gases as $\mathrm{CO}_{2}$ and ethene (synonym: ethylene; $\mathrm{C}_{2} \mathrm{H}_{4}$ ) dissolved in aqueous phase of an in vitro culture system have substantial impact on plant cell metabolism. Both of them, i.e. $\mathrm{CO}_{2}$ and ethene, are continuously generated in plant cells. $\mathrm{CO}_{2}$ acidifies the culture medium and changes its osmomolarity. As one of the plant growth regulators (often called "phytohormones"), ethene causes a variety of metabolic responses of plant cells. Similarly, in the case of $\mathrm{O}_{2}$, its higher concentrations in a culture system have detrimental effects on plant cell biomass resulting in a non-physiological high level of free radicals inside cells. To sum up, all of these remarks indicate the need for further studies on modelling of the growth of biomass in plant cell culture in vitro systems utilising a liquid $\mathrm{PFC}$, and a perfluorinated phase should be taken into consideration as a liquid carrier of $\mathrm{O}_{2}, \mathrm{CO}_{2}$ as well as ethene.

\section{SYMBOLS}

$c$ dimensionless concentration of $\mathrm{O}_{2}$ in aqueous phase

$C_{0} \quad$ relative (dimensionless) initial concentration of the carbon substrate

$C_{O(P F C)} \quad$ molar concentration of $\mathrm{O}_{2}$ in liquid PFC, $\mathrm{mol} \mathrm{dm}^{-3}$

$C_{O(P F C)}^{*}$ molar concentration of $\mathrm{O}_{2}$ in liquid $\mathrm{PFC}$ which is in equilibrium with the $\mathrm{O}_{2}$ partial pressure in gas phase, $\mathrm{mol} \mathrm{dm}^{-3}$

$C_{O(a q)} \quad$ molar concentration of $\mathrm{O}_{2}$ in aqueous phase, $\mathrm{mol} \mathrm{dm}{ }^{-3}$ 
$C_{O(a q)}^{*} \quad$ molar concentration of $\mathrm{O}_{2}$ in aqueous phase which is in equilibrium with the $\mathrm{O}_{2}$ partial pressure in gas phase, $\mathrm{mol} \mathrm{\textrm {dm } ^ { - 3 }}$

$C_{S} \quad$ molar concentration of carbon substrate, $\mathrm{mol} \mathrm{dm}^{-3}$

$C_{S, 0} \quad$ initial concentration of carbon substrate, $\mathrm{mol} \mathrm{dm}^{-3}$

$C_{X} \quad$ mass concentration of biomass, $\mathrm{g} \mathrm{dm}^{-3}$

$C_{X, 0} \quad$ initial mass concentration of biomass, $\mathrm{g} \mathrm{dm}^{-3}$

Da the Damköhler number, Eq. (25)

$H \quad$ the $\mathrm{O}_{2}$ partition coefficient for the water - liquid PFC system

$J \quad$ mass flux transferred between aqueous phase and liquid PFC, $\mathrm{kg} \mathrm{m}^{-2} \mathrm{~s}^{-1}$

$k_{L} a \quad$ volumetric liquid-side mass transfer coefficient, $\mathrm{h}^{-1}$

$K_{m, O} \quad$ saturation constant relative to $\mathrm{O}_{2}, \mathrm{~g} \mathrm{dm}^{-3}$

$K_{m, S} \quad$ saturation constant relative to carbon substrate, $\mathrm{g} \mathrm{dm}^{-3}$

$K_{O} \quad$ dimensionless saturation constant relative to $\mathrm{O}_{2}$

$K_{S} \quad$ dimensionless saturation constant relative to carbon substrate, $\mathrm{g} \mathrm{dm}^{-3}$

$p \quad$ dimensionless concentration of $\mathrm{O}_{2}$ in aqueous phase

$s \quad$ dimensionless concentration of carbon substrate

$s_{0} \quad$ dimensionless initial concentration of carbon substrate

$t \quad$ time, $\mathrm{h}$

$V_{P F C} \quad$ volume of liquid PFC, $\mathrm{dm}^{-3}$

$V_{a q} \quad$ volume of aqueous phase, $\mathrm{dm}^{-3}$

$x \quad$ dimensionless concentration of biomass

$Y_{O X} \quad$ yield of oxygen consumption by unit of biomass coefficient, $\mathrm{mmol} \mathrm{g} \mathrm{g}^{-1}$

$Y_{X S} \quad$ yield of biomass from substrate coefficient, $\mathrm{g} \mathrm{g}^{-1}$

Greek symbols

$\varphi \quad$ the relative volume of aqueous phase

$\mu \quad$ specific growth rate, $\mathrm{h}^{-1}$

$\mu_{\max } \quad$ maximum specific growth rate, $\mathrm{h}^{-1}$

$\tau \quad$ dimensionless time

\section{Superscripts}

* equilibrium with prevailing value in the other phase

BY-2 Nicotiana tabacum cv. BY-2 (cultivar Bright Yellow No. 2 of the tobacco plant)

PFC perfluorochemical (synonym: perfluorocarbon)

$\begin{array}{ll}\text { Subscripts } & \\ 0 & \text { initial } \\ \max & \text { maximum } \\ O & \text { oxygen } \\ P F C & \text { (perfluorinated) phase of liquid PFC } \\ S & \text { carbon substrate } \\ a q & \text { aqueous phase } \\ X & \text { biomass }\end{array}$

\section{REFERENCES}

Amaral P.F.F., Freire M.G., Rocha-Leao M.H.M., Marrucho I.M., Coutinho J.A.P., Coelho M.A.Z., 2007. Optimization of oxygen mass transfer in a multiphase bioreactor with perfluorodecalin as a second liquid phase. Biotechnol. Bioeng., 99, 588-598. DOI: 10.1002/bit.21640.

Davey M.R., Anthony P., Power J.B., Lowe K.C., 2005. Plant protoplast technology: Current status. Acta Physiol. Plant., 27, 117-129. DOI: 10.1007/s11738-005-0044-0. 
Dragoi E. N., Curteanu S., Leon F., Galaction A. I., Cascaval D., 2011. Modeling of oxygen mass transfer in the presence of oxygen-vectors using neural networks developed by differential evolution algorithm. Eng. Appl. Artif. Intel., 24, 1214-1226. DOI: 10.1016/j.engappai.2011.06.004.

Garcia-Ochoa F., Gomez E., Santos V.E., Merchuk J.C., 2010. Oxygen uptake rate in microbial processes: An overview. Biochem. Eng. J., 49, 289-307. DOI: 10.1016/j.bej.2010.01.011.

Hillig F., Pilarek M., Junne S., Neubauer P., 2014. Cultivation of marine microorganisms in single-use systems. Adv. Biochem. Eng. Biotechnol., 138, 179-206. DOI: 10.1007/10_2013_219.

Lowe K.C., 2002. Perfluorochemical respiratory gas carriers: Benefits to cell culture systems. J. Fluor. Chem., 118, 19-26. DOI: 10.1016/S0022-1139(02)00200-2.

Martin M., Montes F.J., Galan M.A., 2010. Mass transfer rates from bubbles in stirred tanks operating with viscous fluids. Chem. Eng. Sci., 65, 3814-3824. DOI: 10.1016/j.ces.2010.03.015.

Ntwampe S.K.O., Williams C.C., Sheldon M. S., 2010. Water-immiscible dissolved oxygen carriers in combination with Pluronic F 68 in bioreactors. Afr. J. Biotechnol., 9, 1106-1114. DOI: 10.5897/AJB09.1007.

Pilarek M., 2014. Liquid perfluorochemicals as flexible and efficient gas carriers applied in bioprocess engineering: and updated overview and future prospects. Chem. Process Eng., 35, 463-487. DOI: 10.2478/cpe2014-0035.

Pilarek M., Grabowska I., Ciemerych M. A., Dąbkowska K., Szewczyk K.W., 2013. Morphology and growth of mammalian cells in a liquid/liquid culture system supported with oxygenated perfluorodecalin. Biotechnol. Lett., 35, 1387-1394. DOI 10.1007/s10529-013-1218-2.

Pilarek M., Grabowska I., Senderek I., Wojasiński M., Janicka J., Janczyk-Ilach K., Ciach T., 2014. Liquid perfluorochemical-supported hybrid cell culture system for proliferation of chondrocytes on fibrous polylactide scaffolds. Bioprocess Biosyst. Eng., 37, 1707-1715. DOI: 10.1007/s00449-014-1143-3.

Pilarek M., Szewczyk K. W., 2008. Effects of perfluorinated oxygen carrier application in yeast, fungi and plant cell suspension cultures. Biochem. Eng. J., 41, 38-42. DOI: 10.1016/j.bej.2008.03.004.

Riess J.G., 2006a. Perfluorocarbon-based oxygen delivery. Artif. Cells Blood Substit. Immobil. Biotechnol., 34, 567-80. DOI: 10.1080/10731190600973824.

van Sonsbeek H.M., van der Tuin S.P., Tramper J., 1992. Mixing in liquid-impelled loop reactors. Biotechnol. Bioeng., 39, 707-716. DOI: 10.1002/bit.260390703.

Suresh S., Srivastava V.C., Mishra I.M., 2009. Techniques for oxygen transfer measurement in bioreactors: a review. J. Chem. Technol. Biotechnol., 84, 1091-1103. DOI: 10.1002/jctb.2154.

Sykłowska-Baranek K., Pilarek M., Cichosz M., Pietrosiuk A., 2014. Liquid perfluorodecalin application for in situ extraction and enhanced naphthoquinones production in Arnebia euchroma cell suspension cultures. Appl. Biochem. Biotechnol., 172, 2618-2627. DOI: 10.1007/s12010-013-0701-5. 